\title{
From generalized Tamari intervals to non-separable planar maps (extended abstract)
}

\author{
Wenjie Fang $1,2 \|$ and Louis-François Préville-Ratelle $\|^{3}$ \\ ${ }^{1}$ IRIF, UMR CNRS 8243, Université Paris Diderot, Paris Cedex 13, France. \\ ${ }^{2}$ LaBRI, UMR CNRS 5800, Université de Bordeaux, Talence, France. \\ ${ }^{3}$ Instituto de Mathemática y Física, Universidad de Talca, 2 norte 685, Talca, Chile.
}

\begin{abstract}
Let $v$ be a grid path made of north and east steps. The lattice $\operatorname{TAM}(v)$, based on all grid paths weakly above the grid path $v$ sharing the same endpoints as $v$, was introduced by Préville-Ratelle and Viennot (2014) and corresponds to the usual Tamari lattice in the case $v=(N E)^{n}$. They showed that $\operatorname{TAM}(v)$ is isomorphic to the dual of $\operatorname{TAM}(\overleftarrow{v})$, where $\overleftarrow{v}$ is the reverse of $v$ with $N$ and $E$ exchanged. Our main contribution is a bijection from intervals in $\operatorname{TAM}(v)$ to non-separable planar maps. It follows that the number of intervals in $\operatorname{TAM}(v)$ over all $v$ of length $n$ is $\frac{2(3 n+3) !}{(n+2) !(2 n+3) !}$. This formula was first obtained by Tutte(1963) for non-separable planar maps.
\end{abstract}

Résumé. Soit $v$ un chemin constitué de pas Nord et Est. Le treillis TAM $(v)$, basé sur tous les chemins faiblement au dessus de $v$ avec les mêmes extrémités que $v$, a été introduit par Préville-Ratelle et Viennot (2014) et correspond au treillis de Tamari classique dans le cas $v=(N E)^{n}$. Ils ont démontré que TAM $(v)$ est isomorphe au treillis dual de $\operatorname{TAM}(\overleftarrow{v})$, où $\overleftarrow{v}$ est $v$ renversé avec $N$ et $E$ échangés. Notre contribution principale est une bijection entre les intervalles de $\operatorname{TAM}(v)$ et les cartes planaires non-séparables. Il s'ensuit que le nombre d'intervalles dans $\operatorname{TAM}(v) \operatorname{sur}$ tous les chemins $v$ de longueur $n$ est donné par $\frac{2(3 n+3) !}{(n+2) !(2 n+3) !}$. Cette formule a été obtenue par Tutte(1963) pour les cartes planaires non-séparables.

Keywords. generalized Tamari interval, non-separable planar map, bijection

\section{Background and main results}

The well-known Tamari lattice can be defined on Dyck paths or some other combinatorial structures counted by Catalan numbers such as binary trees, and it has many connections with several fields, in

\footnotetext{
${ }^{\dagger}$ Email: wenjie.fang@liafa.univ-paris-diderot.fr. W.F. is partially supported by Agence nationale de la Recherche under grant number ANR 12-JS02-001-01 "Cartaplus", and by the City of Paris under the grant "Émergence Paris 2013, Combinatoire à Paris". W.F. acknowledges hospitality from LaBRI, UMR CNRS 5800, Université de Bordeaux, France.

‡Email: preville-ratelled inst-mat . utalca.cl. L.-F. P.-R. is supported by the government of Chile under Proyecto Fondecyt 3140298.
}

1365-8050 (c) 2016 Discrete Mathematics and Theoretical Computer Science (DMTCS), Nancy, France 
particular in algebraic and enumerative combinatorics. In [Cha06], Chapoton showed that intervals in the Tamari lattice are enumerated by the formula

$$
\frac{2}{n(n+1)}\left(\begin{array}{c}
4 n+1 \\
n-1
\end{array}\right)
$$

This formula also counts rooted planar 3-connected triangulations. Many results and conjectures about the diagonal coinvariant spaces of the symmetric group (we refer to [Ber09, Hag08] for further explanation), also called the Garsia-Haiman spaces, led Bergeron to introduce the $m$-Tamari lattice for any integer $m \geq 1$. It was conjectured in [BPR12] and proved in [BMFPR11] and [BMCPR13] that the number of intervals and labeled intervals in the $m$-Tamari lattice of size $n$ are given respectively by the formulas

$$
\frac{m+1}{n(m n+1)}\left(\begin{array}{c}
(m+1)^{2} n+m \\
n-1
\end{array}\right) \text { and }(m+1)^{n}(m n+1)^{n-2} .
$$

These labeled intervals (resp. unlabeled intervals) are conjectured to be enumerated by the same formulas as the dimensions (resp. alternating component) of the trivariate Garsia-Haiman spaces. These connections motivated the introduction of the lattice $\operatorname{TAM}(v)$ in [PRV14] for an arbitrary path $v$ as a further generalization. In particular, the Tamari lattice of size $n$ is given by $\operatorname{TAM}\left((N E)^{n}\right)$, and more generally the $m$-Tamari lattice by $\operatorname{TAM}\left(\left(N E^{m}\right)^{n}\right)$. A precise definition of $\operatorname{TAM}(v)$ will be given in Section 2

The Tamari lattice and its generalizations, while being deeply rooted in algebra, have mysterious enumerative aspects and bijective links yet to be unearthed. For instance, intervals in the Tamari lattice are equi-enumerated with planar triangulations, and a bijection was given by Bernardi and Bonichon in [BB09]. Similarly, the numbers of usual and labeled intervals in the $m$-Tamari lattice in [BMFPR11] and [BMCPR13] are also given by simple planar-map-like formulas, where a combinatorial explanation is still missing. In this context, similar to the bijection in [BB09], we also discover a bijection between intervals and maps, contributing to the combinatorial understanding of the Tamari lattice.

Our main contribution is a bijection between intervals in generalized Tamari lattices and non-separable planar maps. To describe it, we need two intermediate structures: one called synchronized interval, which is a special kind of intervals in the usual Tamari lattice; the other called decorated tree, basically a kind of rooted trees with labels on their leaves that satisfy certain conditions. The bijection from generalized Tamari intervals to synchronized intervals is implicitly given in [PRV14]. We then show that an exploration process gives a bijection between non-separable planar maps and decorated trees, and there is a bijection between decorated trees and synchronized intervals.

As a consequence of our bijection, we give the following enumeration formula of intervals in $\operatorname{TAM}(v)$.

Theorem 1.1 The total number of intervals in $\operatorname{TAM}(v)$ over all possible $v$ of length $n$ is given by

$$
\sum_{v \in(N, E)^{n}} \operatorname{Int}(\operatorname{TAM}(v))=\frac{2(3 n+3) !}{(n+2) !(2 n+3) !} .
$$

We also give a proof using generating functions. Various other combinatorial structures are also enumerated by (1), including non-separable planar maps [Tut63], 2-stack sortable permutations, non-separable permutations and $\beta-(1,0)$ trees ( $c f$. Chapter 2 of [Kit11]). This enumeration formula was first obtained by Tutte in [Tut63] for non-separable planar maps. 
Our bijection also unveils a surprising appearance of map duality in generalized Tamari lattices. For a word $v$ in $N, E$, let $\overleftarrow{v}$ be the word obtained from exchanging $N$ and $E$ in the reverse of $v$. It was proved in [PRV14] that $\operatorname{TAM}(v)$ is isomorphic to the dual of $\operatorname{TAM}(\overleftarrow{v})$. This isomorphism is related to map duality by the following result, whose proof by recursive constructions of both objects is omitted here.

Theorem 1.2 The isomorphism from $\operatorname{TAM}(v)$ to $\operatorname{TAM}(\overleftarrow{v})$ applied on generalized Tamari intervals is equivalent to map duality under our bijection.

\section{From canopy intervals to synchronized intervals}

A grid path is a finite walk on the square grid, starting at $(0,0)$ and consisting of north and east unit steps denoted by $N$ and $E$ respectively. The size of a grid path is the number of steps it has. For any grid path $v$, let $\operatorname{TAM}(v)$ be the set of grid paths that are weakly above $v$ and share the same endpoints as $v$. The covering relation defined as follows gives $\operatorname{TAM}(v)$ a lattice structure. For a grid point $p$, we define the horizontal distance horiz $z_{v}(p)$ to be the maximum number of east steps that we can take starting from $p$ without crossing $v$. The left part of Figure 1 shows an example of a grid path in $\operatorname{TAM}(v)$, with the horizontal distance of its grid points. Suppose that $p$ is a grid point on $v_{1}$ preceded by a step $E$ and followed by a step $N$. Let $p^{\prime}$ be the first grid point on $v_{1}$ after $p$ such that $\operatorname{horiz}_{v}\left(p^{\prime}\right)=\operatorname{horiz}_{v}(p)$, and $v_{1}\left[p, p^{\prime}\right]$ the subpath of $v_{1}$ from $p$ to $p^{\prime}$. By switching the step $E$ just before $p$ and the subpath $v_{1}\left[p, p^{\prime}\right]$ in $v_{1}$, we obtain another grid path $v_{1}^{\prime}$ in $\operatorname{TAM}(v)$. The covering relation $\prec_{v}$ in $\operatorname{TAM}(v)$ is given by $v_{1} \prec_{v} v_{1}^{\prime}$, and its transitive closure $\leq_{v}$ gives $\operatorname{TAM}(v)$ a lattice structure. The right part of Figure 1 shows an example of $\prec_{v}$. The grid path $v$ is called the canopy due to what it represents on binary trees, and it is also the minimal element in $\operatorname{TAM}(v)$. A canopy interval of size $n \geq 0$ is given by a triple of grid paths $\left(v_{2}, v_{1}, v\right)$, all of size $n$, such that $v_{1} \leq_{v} v_{2}$ in $\operatorname{TAM}(v)$. They are in bijection with a family of intervals in the usual Tamari lattice called synchronized intervals, which are better suited for establishing the bijections in the next sections. We refer readers to [PRV14] for more details about TAM $(v)$ and canopy intervals.

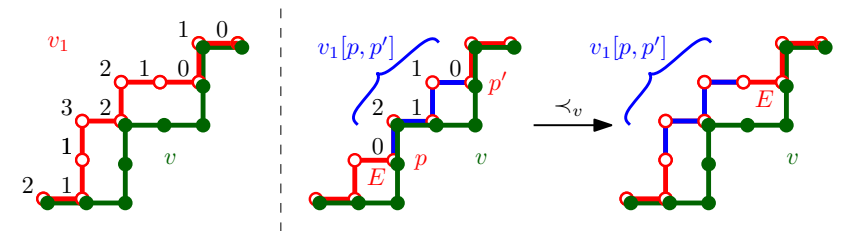

Fig. 1: Example of horizontal distances and the covering relation in $\operatorname{TAM}(v)$

From now on, we only work in the usual Tamari lattice formed by Dyck paths. A Dyck path of size $n$ is a finite walk on $\mathbb{Z}^{2}$, starting at $(0,0)$, consisting of $n$ up steps $u=(1,1)$ and $n$ down steps $d=(1,-1)$, and never falling below the $x$-axis. We choose to use diagonal steps $(u, d)$ for Dyck paths instead of north and east steps $(N, E)$ of grid paths to underline that these two types of paths are elements in different lattices. It is known (cf. [DV84, Lev59]) that pairs of non-crossing grid paths with the same endpoints of size $n-1 \geq 0$ are counted by Catalan number $C_{n}=\frac{1}{n+1}\left(\begin{array}{c}2 n \\ n\end{array}\right)$, which also counts Dyck paths of size $n$.

For a Dyck path $P=\left(p_{i}\right)_{1 \leq i \leq 2 n}$ where $p_{i}$ are steps, let $i_{1}, \ldots, i_{n}$ be the indices such that $p_{i_{k}}=u$. We define Type $(P)$ as the following word $w$ of length $n-1$ : for $k \leq n-1$, if $p_{i_{k}}=p_{i_{k}+1}=u$, then $w_{k}=E$, otherwise $w_{k}=N$. Let $I(v)$ be the set of Dyck paths of type $v$. It is also an interval in the usual Tamari lattice. Types of Dyck paths are related to $\operatorname{TAM}(v)$ by the following theorem. 
Theorem 2.1 (Theorem 3 in [TPR14]) The usual Tamari lattice is partitioned into intervals $I(v) \simeq$ $\operatorname{TAM}(v)$ of Dyck paths of all $2^{n-1}$ possible type $v$.
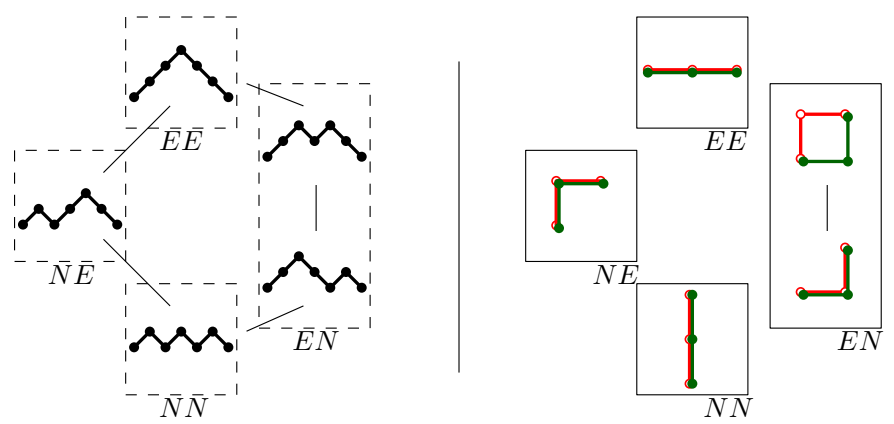

Fig. 2: Partition of the usual Tamari lattice of size 3, and the corresponding generalized Tamari intervals

An example of this partition by type can be seen in Figure 2. A synchronized interval is an interval in an $I(v)$ for some grid path $v$. Given a grid path $v$, the explicit bijection in [PRV14] between pairs of non-crossing grid paths of size $n-1$ and Dyck paths of size $n$ specializes to a lattice isomorphism between $\operatorname{TAm}(v)$ and the interval of the Tamari lattice made of Dyck paths of type $v$, which induces a bijection between their intervals. We recall that the size of a canopy interval is the number of steps, while the size of a synchronized interval is the number of up steps. We thus have a bijection between canopy intervals of size $n-1$ and synchronized intervals of size $n$, which implies that they are equi-enumerated.

\section{Recursive decompositions}

We are now interested in the link between two families of objects: synchronized intervals in the usual Tamari lattice on Dyck paths of size $n$, and non-separable planar maps with $n+1$ edges. In fact, their enumeration is governed by the same functional equation. In this section, we show how to decompose recursively these two families of combinatorial objects. We reiterate that our main contribution, which is a non-recursive bijection, will be described explicitly in the next section.

\subsection{Recursive decomposition of synchronized intervals}

We define a properly pointed Dyck path to be a Dyck path $P=P_{\ell} P_{r}$ such that $P_{\ell}$ and $P_{r}$ are Dyck paths, and $P_{\ell}$ is not empty unless $P$ is itself empty. A properly pointed synchronized interval $\left[P^{\ell} P^{r}, Q\right]$ is a synchronized interval where the lower Dyck path is properly pointed. We denote by $\mathcal{I}_{n}$ the set of synchronized intervals of size $n$, and by $\mathcal{I}_{n}^{\bullet}$ the set of properly pointed synchronized intervals of size $n$. We state the following construction similar to Proposition 7 in [BMCPR13].

Proposition 3.1 Let $I_{1}=\left[P_{1}^{\ell} P_{1}^{r}, Q_{1}\right]$ be a properly pointed synchronized interval and $I_{2}=\left[P_{2}, Q_{2}\right]$ a synchronized interval. We construct the Dyck paths

$$
P=u P_{1}^{\ell} d P_{1}^{r} P_{2}, \quad Q=u Q_{1} d Q_{2} .
$$

Then $I=[P, Q]$ is a synchronized interval. Moreover, this transformation from $\left(I_{1}, I_{2}\right)$ to $I$ is a bijection between $\cup_{n \geq 0} \mathcal{I}_{n}^{\bullet} \times \cup_{n \geq 0} \mathcal{I}_{n}$ and $\cup_{n>0} \mathcal{I}_{n}$. 


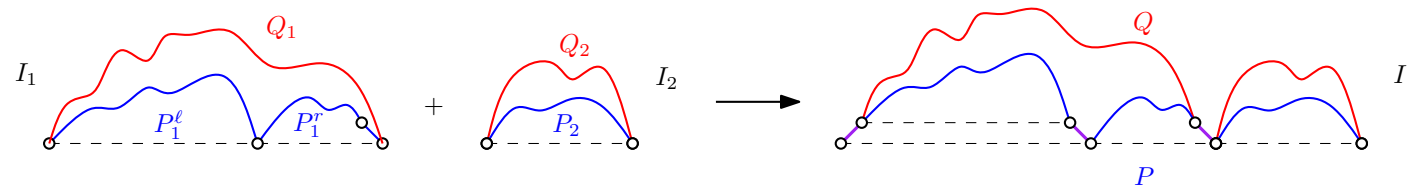

Fig. 3: Composition of synchronized intervals

A contact of a Dyck path $P$ is an intersection of $P$ with the $x$-axis, including both endpoints. Let $\operatorname{cont}(P)$ be the number of contacts of $P$. We define $F(x, t)$ as the following generating function of synchronized intervals:

$$
F(x, t)=\sum_{n \geq 1} \sum_{[P, Q] \in \mathcal{I}_{n}} t^{n} x^{\operatorname{cont}(P)-1} .
$$

In Proposition 3.1, the generating functions of the intervals of the form $\left[u P_{1}^{\ell} d P_{1}^{r}, u Q_{1} d\right]$ is given by $x t\left(1+\frac{F(x, t)-F(1, t)}{x-1}\right)$, where the divided difference accounts for lifting each non-initial contact (individually) over all elements in $\mathcal{I}_{n}$. We observe that the path $P=u P_{1}^{\ell} d P_{1}^{r} P_{2}$ has $\operatorname{cont}(P)-1=$ $\left(\operatorname{cont}\left(u P_{1}^{\ell} d P_{1}^{r}\right)-1\right)+\left(\operatorname{cont}\left(P_{2}\right)-1\right)$. Therefore, from Proposition 3.1. we obtain the functional equation

$$
F(x, t)=x t(1+F(x, t))\left(1+\frac{F(x, t)-F(1, t)}{x-1}\right) .
$$

\subsection{Recursive decomposition of non-separable planar maps}

We now turn to non-separable planar maps, which were first enumerated by Tutte in [Tut63] using algebraic methods, then by Jacquard and Schaeffer in [JS98] using a bijection based on a recursive decomposition. A planar map is an embedding of a connected graph on the sphere defined up to homeomorphism, with one oriented edge called the root. The origin vertex of the root is called the root vertex. We call the face on the left of the root the outer face. A planar map is called separable if its edges can be partitioned into two sets such that only one vertex $v$ is adjacent to some edges in both sets. Such a vertex is called a cut vertex. A non-separable planar map is a planar map containing at least two edges that is not separable. Figure 4 gives an example of such a map. Note that we exclude the two one-edge maps.
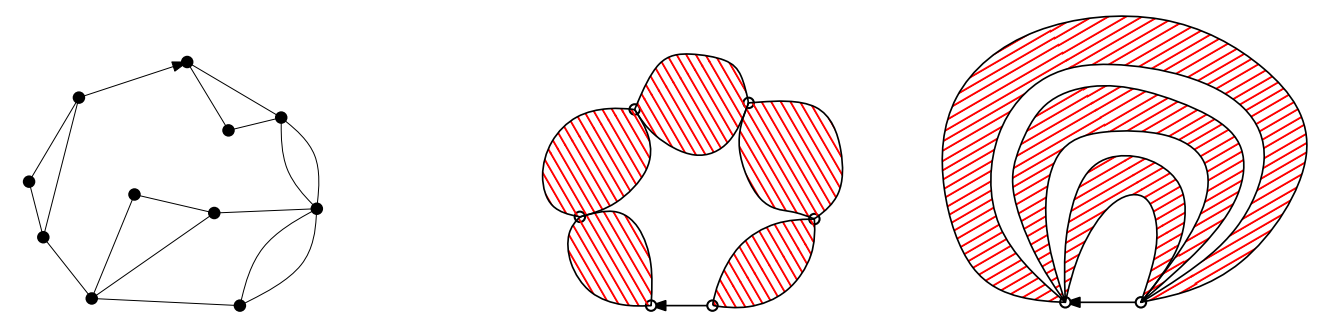

Fig. 4: A non-separable planar map, and series/parallel decompositions of non-separable planar maps

Proposition 3.2 (Corollary II in [Tut63]) The dual of a non-separable planar map is also non-separable. 
There are two ways to decompose non-separable planar maps recursively, which are dual to each other. We will call them "series" and "parallel" decompositions respectively. We only need one decomposition for the functional equation, but describing both leads to a more thorough understanding. In Figure 4 , we illustrate how larger maps are built from smaller maps in both series and parallel ways.

For the series decomposition of a non-separable planar map $M$, we delete its root, and the remaining map $M^{\prime}$ may cease to be non-separable. Every cut vertex thus splits $M^{\prime}$ into two parts, each containing an endpoint of the root. The remaining part is thus a series of non-separable components (and possibly single edges) linked by vertices (see Figure 4 , middle). Let $\mathcal{M}_{n}$ be the set of non-separable planar maps with $n+1$ edges, and $M_{s}(x, t)$ the generating function of non-separable planar maps defined as

$$
M_{s}(x, t)=\sum_{n \geq 1} \sum_{M \in \mathcal{M}_{n}} t^{n} x^{\operatorname{deg}(\text { outer face })-1} .
$$

For a component in the series, we root it at its first edge adjacent to the outer face in clockwise order to obtain a non-separable planar map such that the root vertex is one of the linking vertices in the chain. Inversely, from a non-separable planar map with $n+1$ edges and outer face of degree $k+1$ (therefore of contribution $t^{n} x^{k}$ in $M_{s}(x, t)$ ), there are $k$ choices for the linking vertex other than the root vertex to obtain a component, each adding a value from 1 to $k$ to the outer face degree. These choices thus have a total contribution of $t^{n+1} \sum_{i=1}^{k} x^{i}=t x \frac{t^{n} x^{k}-t^{n}}{x-1}$. Therefore, by the series decomposition we have

$$
M_{s}(x, t)=\frac{x t+x t \frac{M_{s}(x, t)-M_{s}(1, t)}{x-1}}{1-\left(x t+x t \frac{M_{s}(x, t)-M_{s}(1, t)}{x-1}\right)} .
$$

A reordering gives the same functional equation as (2).

For the parallel decomposition, we consider the effect of contracting the root. Let $u$ be the vertex of the map $M^{\prime}$ obtained from the contraction of the root. The only possible cut vertex in $M^{\prime}$ is $u$. By splitting $u$, we have an ordered list of non-separable planar maps (and possibly loops) that come in parallel (see Figure 4 right). Let $M_{p}(x, t)$ be the following generating function of non-separable planar maps, where $v$ is the root vertex of $M$ :

$$
M_{p}(x, t)=\sum_{M \in \mathcal{M}_{n}} t^{n} x^{\operatorname{deg}(v)-1} .
$$

We leave readers to check that the parallel decomposition leads to the same equation as $M_{s}$. Since $F, M_{s}, M_{p}$ all obey the same functional equation, we have $F=M_{s}=M_{p}$, therefore these objects are equi-enumerated under the specified statistics, which invites us to search for a bijective proof. Observe that $M_{s}=M_{p}$ can easily be explained by a suitable map duality that swaps the corresponding statistics.

\section{Bijections}

We now present our main contribution. To describe our bijection from synchronized intervals to nonseparable planar maps, we first introduce a family of trees. We take the convention that the root of a tree is of depth 0 . The traversal order on the leaves of a tree is simply the left-to-right order. A decorated tree is a rooted plane tree with an integer label at least -1 on each leaf, satisfying the following conditions:

1. For a leaf $\ell$ adjacent to a vertex of depth $p$, the label of $\ell$ is strictly smaller than $p$. 
2. For each internal node of depth $p>0$, there is at least one leaf in its descendants with a label at most $p-2$.

3. For $t$ a node of depth $p$ and $T^{\prime}$ a subtree rooted at a child of $t$, consider leaves of $T^{\prime}$ in traversal order. If a leaf $\ell$ is labeled $p$ (which is the depth of $t$ ), each leaf in $T^{\prime}$ coming before $\ell$ has a label at least $p$.

The right side of Figure 5 shows an example of a decorated tree. In a decorated tree, a leaf labeled with -1 is called a free leaf. We denote by $\mathcal{T}_{n}$ the set of decorated trees with $n$ edges (internal and external).

\subsection{From maps to trees}

We start with a bijection from non-separable planar maps to decorated trees which relies on the following exploration procedure. For a non-separable planar map $M$ with its root pointing from $v$ to $u$, we perform a depth-first exploration of vertices in clockwise order around each vertex, starting from $v$ and the root. When the exploration along an edge encounters an already-visited vertex $x$, we attach a leaf labeled with the depth of $x$ in the tree, with the convention that the depth of $v$ is -1 . Since the map is non-separable, this exploration gives a spanning tree whose root $v$ has degree 1 . We then delete the edge $(v, u)$ to obtain $\mathrm{T}(M)$. Figure 5 shows an instance of the transformation $\mathrm{T}$.
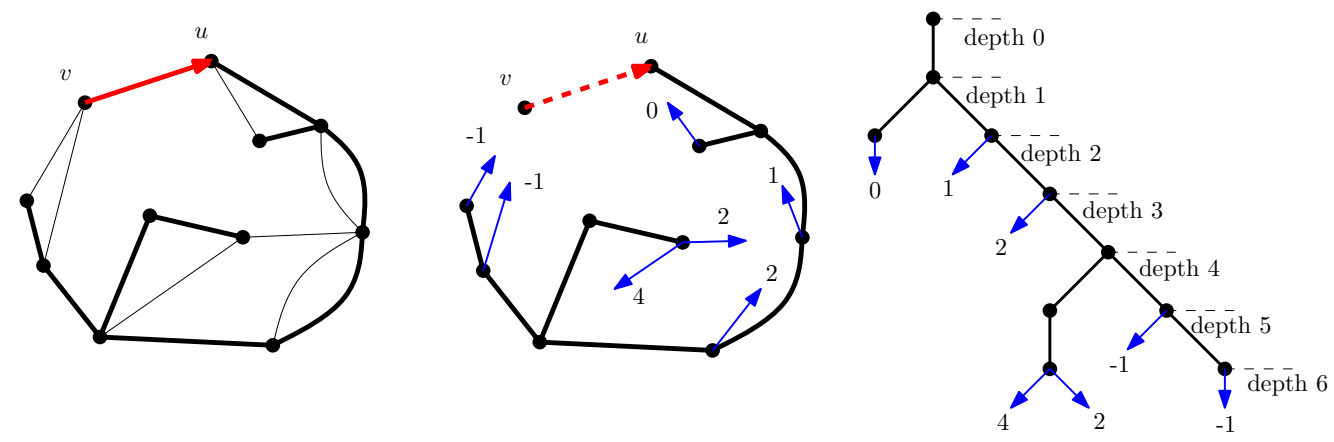

Fig. 5: An example of the bijection between non-separable planar maps and decorated trees

By abuse of notation, we identify internal nodes of $\mathrm{T}(M)$ with corresponding vertices in $M$. We notice that, for children of the same vertex in the tree, the ones being visited first in the map come last in the traversal order. Readers familiar with graph algorithms will notice that this exploration procedure is very close to an algorithm proposed by Hopcroft and Tarjan in [HT73] that finds 2-connected components of an undirected graph. Indeed, our exploration procedure can be seen as an adaptation of that algorithm in the case of planar maps.

We now present the inverse $\mathrm{S}$ of $\mathrm{T}$. For a decorated tree $T$ rooted at $u$, we define $\mathrm{S}(T)$ as the map obtained according to the following steps.

1. Attach an edge $\{u, v\}$ to $u$ with a new vertex $v$, and make it the root, pointing from $v$ to $u$.

2. In clockwise order, for each leaf $\ell$ in the tree starting from the last leaf in traversal order, do the following. Let $t$ be the parent of $\ell$ and $p$ the label of $\ell$. Let $s$ be the ancestor of $\ell$ of depth $p$, and $e$ 
the first edge of the path from $s$ to $\ell$ (thus an edge adjacent to $s$ ). We replace $\ell$ by an edge from $t$ to $s$, starting at the position of $\ell$ at $t$ and ending just after $e$ in clockwise order around $s$.

From the definitions of $\mathrm{S}$ and $\mathrm{T}$, we can see that the first condition of decorated trees guarantees that it is an exploration tree of a certain map, the second guarantees that the map is non-separable, and the third guarantees that the map is planar. We now prove that $\mathrm{T}$ and $\mathrm{S}$ are well-defined transformations between $\mathcal{M}$ and $\mathcal{T}$, that they are in fact bijective and are the inverse of each other.

Proposition 4.1 $\mathrm{T}\left(\mathcal{M}_{n}\right) \subset \mathcal{T}_{n}$, and $\mathrm{S}\left(\mathcal{T}_{n}\right) \subset \mathcal{M}_{n}$.

Proof (Sketch): The first part is clear. For the second part, it is clear that S preserves the size $n$ and the resulting map is planar. Let $T$ be a decorated tree, if there is a cut vertex $t$ in $\mathrm{S}(T)$, by construction $t \neq v$, and as a vertex of $T$, the vertex $t$ must violate the second condition of decorated trees, which is impossible. Therefore, $\mathrm{S}(T)$ is non-separable.

Proposition 4.2 For any non-separable planar map $M$, we have $\mathrm{S}(\mathrm{T}(M))=M$.

Proof (Sketch): Using leaf labels, it is clear from the definitions that $\mathrm{S}(\mathrm{T}(M))$ is equal to $M$ as a graph, and we only need to show that they have the same cyclic order of edges around each vertex. Let $t$ be an internal node of depth $p$ in $\mathrm{T}(M)$. We consider its descendant leaves with label $p$ in one of its subtrees $T^{\prime}$ induced by a descendant edge $e$ adjacent to $t$. Let $\ell_{i}$ be such a leaf. When reconnecting, the new edge should come before $e$ by the construction of $\mathrm{T}(M)$, and it cannot encompass other subtrees rooted at a child of $t$, or else $t$ will be a cut vertex (see the left part of Figure 6). If there are multiple such leaves in $T^{\prime}$, their order is fixed by planarity (see the right part of Figure 6). The reasoning also works for the extra vertex $v$ that is not in $\mathrm{T}(M)$. Thus, there is only one way to recover a non-separable planar map from $\mathrm{T}(M)$, and we have $\mathrm{S}(\mathrm{T}(M))=M$.

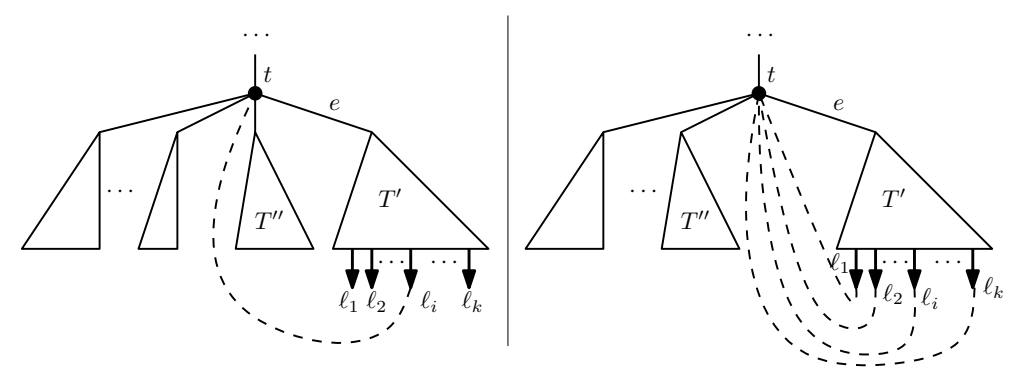

Fig. 6: Illustration of the proof of Proposition 4.2

Proposition 4.3 For any decorated tree $T$, we have $\mathrm{T}(\mathrm{S}(T))=T$.

Proof: Let $M=\mathrm{S}(T)$. We only need to show that the exploration tree $T^{\prime}$ of $M$ is $T$ without labels. Closing each leaf one by one in the procedure $\mathrm{S}(T)$, it is clear that the exploration tree at each step is the same, therefore $T^{\prime}=T$.

Theorem 4.4 The transformation $\mathrm{T}$ is a bijection from the set of non-separable planar maps $\mathcal{M}_{n}$ to the set of decorated trees $\mathcal{T}_{n}$, and $\mathrm{S}$ is its inverse. 


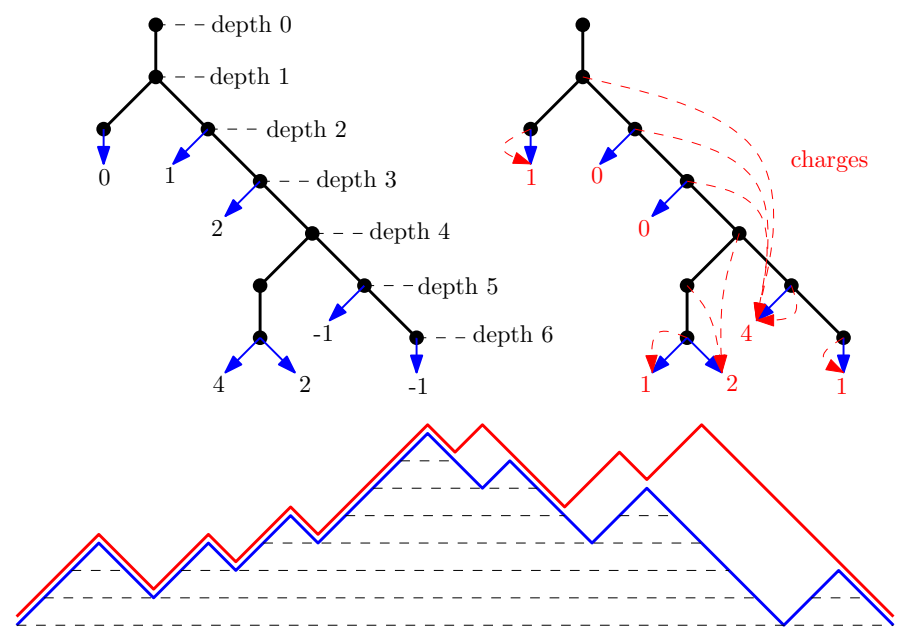

Fig. 7: An example of a decorated tree, the charges on its leaves and the corresponding synchronized interval

\subsection{From trees to intervals}

We now construct a bijection from decorated trees to synchronized intervals. For a decorated tree $T$, we want to construct a synchronized interval $[\mathrm{P}(T), \mathrm{Q}(T)]$. For the upper path, we simply define $\mathrm{Q}(T)$ as the transformation from the tree $T$ to a Dyck path by taking the depth evolution in the tree traversal. The definition of $\mathrm{P}$ is more complicated. We need to define a quantity on leaves of the tree $T$ called the charge. The transformation $P$ takes the following steps.

1. Every leaf has an initial charge 0 . For each internal vertex $v$ of depth $p>0$, we add 1 to the charge of the first leaf in its descendants (in traversal order) with a label at most $p-2$.

2. We perform a traversal of the tree in order to construct a word in $u, d$. When we first visit an internal edge, we append $u$ to the word. When we first visit a leaf with charge $k$, we append $u d^{k+1}$ to the word. We thus obtain the word $\mathrm{P}(T)$.

An example of the whole process is given in Figure 7 Before proving that this construction is welldefined and gives a synchronized interval, we need to introduce some additional notation and to borrow a lemma from [BMFPR11.

There is a natural matching between up steps and down steps in a Dyck path defined as follows: let $u_{i}$ be an up step of a Dyck path $P$, we draw a horizontal ray from the middle of $u_{i}$ to the right until it meets a down step $d_{j}$, and we say that $u_{i}$ is matched with $d_{j}$. We denote by $\ell_{P}\left(u_{i}\right)$ the distance from $u_{i}$ to $d_{j}$ in $P$ considered as a word. We define the function $D_{P}$ by $D_{P}(i)=\ell_{P}\left(u_{i}\right)$, where $u_{i}$ is the $i^{\text {th }}$ up step in $P$.

Lemma 4.5 (Proposition 5 in [BMFPR11]) Let $P$ and $Q$ be two Dyck paths of size $n$. Then $P \leq Q$ in the Tamari lattice if and only if $D_{P}(i) \leq D_{Q}(i)$ for all $1 \leq i \leq n$.

We now prove that the bijections $\mathrm{P}$ and $\mathrm{Q}$ send a decorated tree to a synchronized interval.

Proposition 4.6 For a decorated tree $T$, the paths $\mathrm{P}(T)$ and $\mathrm{Q}(T)$ are Dyck paths, and $[\mathrm{P}(T), \mathrm{Q}(T)]$ is a synchronized interval. 
Proof (Sketch): Since $\mathrm{Q}(T)$ is the depth evolution of the traversal of $T$, it is a well-defined Dyck path. By the charging process, it is clear that $\mathrm{P}(T)$ is of the same length as $\mathrm{Q}(T)$, starting and ending on the $x$-axis, and we need to show that it is positive. When we look at the traversal construction of $\mathrm{P}(T)$, for each nonroot internal node $w$, the edge linking $w$ to its parent is always visited before the leaf charged by $w$, which is a descendant of $w$. Therefore, $\mathrm{P}(T)$ is a Dyck path. We also see that Type $(\mathrm{P}(T))=\operatorname{Type}(\mathrm{Q}(T))$, since in both $\mathrm{P}(T)$ and $\mathrm{Q}(T)$, a letter $u$ is followed by a letter $d$ if and only if it corresponds to a leaf.

We now need to show that $[\mathrm{P}(T), \mathrm{Q}(T)]$ is a Tamari interval. Let $v$ be a non-root internal node, which corresponds to an up step $u_{i}$ in $P$, and $\ell$ the leaf charged by $v$. The main idea is to show that the matching down step $d_{i}$ of $u_{i}$ in $P$ is produced by $\ell$, which guarantees the condition in Lemma 4.5 since $\ell$ is a descendant leaf of $v$. This is proved by a technical analysis on the charging process, which shows that the leaves corresponding to the down steps between $u_{i}$ and $d_{i}$ in $P$ are charged by descendants of $v$, using the minimality of the position of $\ell$ in the charging process of $v$.

We now describe the inverse transformation $\mathrm{R}$, which sends a synchronized interval $[P, Q]$ to a decorated tree $T=\mathrm{R}([P, Q])$ by the following steps.

1. We construct the tree structure of $T$ from $Q$.

2. We perform the following on each leaf. Let $\ell$ be a leaf. Suppose that $\ell$ gives rise to the $i^{\text {th }}$ up step in $Q$. We look at the lowest point of the consecutive down steps that comes after the $i^{\text {th }}$ up step in $P$, and we draw a ray to the left until intersecting the midpoint of two consecutive up steps in $P$. Suppose that the lower up step is the $j^{\text {th }}$ up step in $P$. We take $e$ the edge in $T$ that gives rise to the $j^{\text {th }}$ up step in $Q$. Let $p$ be the depth of the shallower end point of $e$. We label the leaf $\ell$ with $p$. In the case that no such intersection exists, $\ell$ is labeled -1 . Figure 8 shows an example of this procedure for a leaf.

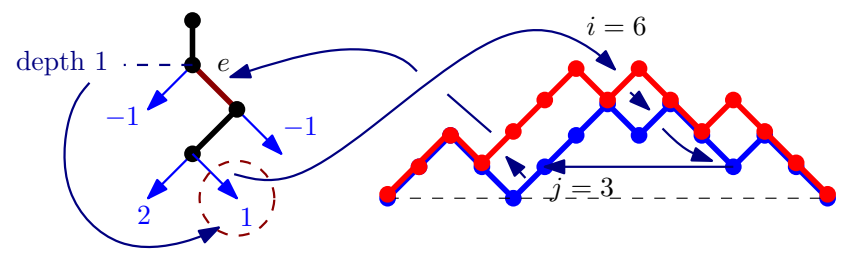

Fig. 8: An example of how to recover leaf labels using the lower path $P$ (here, leaf with label 1)

Proposition 4.7 For $[P, Q]$ a synchronized interval, the tree $T=\mathrm{R}([P, Q])$ is a decorated tree.

Proof (Sketch): We need to verify that $T$ satisfies the three conditions of decorated trees. The first one comes from the definition of R. For the second one, let $v$ be a non-root internal node, $p$ the depth of $v$, and $e$ the edge linking $v$ to its parent. Suppose that the $i^{\text {th }}$ up step in $Q$ is given by the first visit to $e$. Let $d_{i}$ be the matching down step of $i^{\text {th }}$ up step in $P$. We can show by a detailed analysis that the leaf $\ell$ producing $d_{i}$ satisfies the second condition. The third condition can be proved by showing that, for a leaf $\ell$, any leaf that breaks the condition for $\ell$ also breaks the horizontal ray in the definition of $\mathrm{R}$, which is impossible. 
Proposition 4.8 Let $[P, Q]$ be a synchronized interval and $T=\mathrm{R}([P, Q])$, we have $\mathrm{P}(T)=P$ and $\mathrm{Q}(T)=Q$.

Proof (Sketch): For $Q$ it is clear. For $P$, given the path $Q$ and the condition that $[\mathrm{P}(T), \mathrm{Q}(T)]$ is a synchronized interval, $\mathrm{P}(T)$ is totally determined by the charge of each leaf. We only need to show that each leaf in $T$ receives the correct amount of charges. This is done by a detailed analysis of the charging process. Let $\ell$ be a leaf in $T$. We show that each down step $d_{i}$ in the maximal consecutive down steps of $\ell$ in $P$, except the highest one, corresponds bijectively to an internal node $v$ of $T$ that charges $\ell$, which makes the correct number of charges.

Proposition 4.9 Let $T_{1}, T_{2}$ be two decorated trees. If $\mathrm{P}\left(T_{1}\right)=\mathrm{P}\left(T_{2}\right)$ and $\mathrm{Q}\left(T_{1}\right)=\mathrm{Q}\left(T_{2}\right)$, then $T_{1}=$ $T_{2}$.

Proof: Suppose that $T_{1} \neq T_{2}$. $T_{1}$ and $T_{2}$ only differ on labels since $\mathrm{Q}\left(T_{1}\right)=\mathrm{Q}\left(T_{2}\right)$. Let $\ell$ be the first leaf in the traversal order where $T_{1}$ and $T_{2}$ differ, with label $k_{1}$ in $T_{1}$, label $k_{2}$ in $T_{2}$, and $k_{1}>k_{2}$. Nodes that charge $\ell$ in $T_{1}$ also charge $\ell$ in $T_{2}$. Since $\mathrm{P}\left(T_{1}\right)=\mathrm{P}\left(T_{2}\right)$, we know that $\ell$ has the same charges in $T_{1}$ and $T_{2}$, thus is also charged by the same set of nodes. Let $u$ be the ancestor of $\ell$ of depth $k_{1}+1$, which exists due to the definition of decorated trees. Since $u$ does not charge $\ell$ in $T_{1}$, thus in $T_{2}$ neither. By the charging process, in $T_{2}$ there must be a leaf $\ell^{\prime}$ in the subtree induced by $u$ with a label at most $k_{2}$ that comes before $\ell$. The minimality of $\ell$ implies that $\ell^{\prime}$ also has a label $k_{2}$ in $T_{1}$, violating the third condition of decorated trees, which is impossible.

Theorem 4.10 The transformation $\mathrm{R}$ is a bijection from $\mathcal{I}_{n}$ to $\mathcal{T}_{n}$ for all $n \geq 1$, with $[\mathrm{P}, \mathrm{Q}]$ its inverse.

By composing the two bijections $\mathrm{T}$ and $[P, Q]$, we obtain a natural bijection from non-separable planar maps with $n+2$ edges to canopy intervals of size $n$ via decorated trees, therefore these two kinds of objects are enumerated by the same formula.

Remark 1 There is a version of parallel decomposition of non-separable planar maps that just removes one non-separable component in the rightmost part of Figure. 4. This decomposition is isomorphic to the one underlying Proposition 3.1 for synchronized intervals. Thus, there exists a "canonical", recursively defined bijection between $\mathcal{M}_{n}$ and $\mathcal{I}_{n}$. We can prove that it coincides with our bijection $[\mathrm{P}, \mathrm{Q}] \circ \mathrm{T}$.

\section{Discussion}

Our bijection allows us not only to enumerate canopy intervals, but also to investigate various statistics in these two kinds of objects, both coming with interesting hidden symmetries. It is also interesting to ask for significant statistics that are also transferred by our bijection and other interesting natural involutions.

We know that $\operatorname{TAM}\left((N E)^{n}\right)$ is isomorphic to the usual Tamari lattice, and in [BB09], Bernardi and Bonichon gave a bijection between Tamari intervals and planar rooted triangulations. It is thus natural to look for a similar bijection as a specialization of our bijection, and eventually a generalization to $\operatorname{TAM}\left(\left(N E^{m}\right)^{n}\right)$, which is isomorphic to the $m$-Tamari lattice.

As a final remark, in Chapter 2 of [Kit11], there is a sequence of bijections that starts with 2-stacks sortable permutations and ends with non-separable planar maps, which goes through 8 intermediate families of objects. It would be interesting to see how our work is related to these equi-enumerated objects. 


\section{Acknowledgement}

The two authors would like to thank Mireille Bousquet-Mélou, Guillaume Chapuy, Luc Lapointe and

Xavier Viennot for many fruitful and inspiring discussions.

\section{References}

[BB09] O. Bernardi and N. Bonichon. Intervals in Catalan lattices and realizers of triangulations. J. Combin. Theory Ser. A, 116(1):55-75, 2009.

[Ber09] François Bergeron. Algebraic combinatorics and coinvariant spaces. CMS Treatises in Mathematics. Canadian Mathematical Society, Ottawa, ON, 2009.

[BMCPR13] M. Bousquet-Mélou, G. Chapuy, and L.-F. Préville-Ratelle. The representation of the symmetric group on m-Tamari intervals. Adv. Math., 247:309-342, 2013.

[BMFPR11] M. Bousquet-Mélou, É. Fusy, and L.-F. Préville-Ratelle. The number of intervals in the $m$ Tamari lattices. Electron. J. Combin., 18(2):Research Paper 31, 26 pp. (electronic), 2011.

[BPR12] François Bergeron and Louis-François Préville-Ratelle. Higher trivariate diagonal harmonics via generalized Tamari posets. J. Comb., 3(3):317-341, 2012.

[Cha06] F. Chapoton. Sur le nombre d'intervalles dans les treillis de Tamari. Sém. Lothar. Combin., pages Art. B55f, 18 pp. (electronic), 2006.

[DV84] M. Delest and X.G. Viennot. Algebraic languages and polyominos enumeration. Theor. Comput. Sci., 34:169-206, 1984.

[Hag08] J. Haglund. The q,t-Catalan numbers and the space of diagonal harmonics, volume 41 of University Lecture Series. American Mathematical Society, Providence, RI, 2008.

[HT73] J. Hopcroft and R. Tarjan. Algorithm 447: Efficient algorithms for graph manipulation. Commun. ACM, 16(6):372-378, 1973.

[JS98] B. Jacquard and G. Schaeffer. A bijective census of nonseparable planar maps. J. Combin. Theory Ser. A, 83(1):1-20, 1998.

[Kit11] S. Kitaev. Patterns in permutations and words. Monographs in Theoretical Computer Science. An EATCS Series. Springer, Heidelberg, 2011.

[Lev59] J. Levine. Note on the number of pairs of non-intersecting routes. Scripta Math., 24:335$338,1959$.

[PRV14] L.-F. Préville-Ratelle and X. Viennot. An extension of Tamari lattices. In Proceedings of 27th International Conference on Formal Power Series and Algebraic Combinatorics (FPSAC 2015), 2014. arXiv preprint arXiv:1406.3787.

[Tut63] W. T. Tutte. A census of planar maps. Canad. J. Math, 15(2):249-271, 1963. 